Research Article

www.jestr.org

\title{
A New Method of Designing Electrical Impedance Matching Network for Piezoelectric Ultrasound Transducer
}

\author{
Jianfei An* and Shuangxi Zhang \\ Dept. of Modern Physics, University of Science and Technology of China, Hefei, Anhui-China
}

Received 10 October 2013; Accepted 22 January 2014

\begin{abstract}
A new method that is based on genetic algorithm (GA) is developed to design electrical impedance matching network for broadband piezoelectric ultrasound transducer. The new method can both optimize the topology of the matching network and perform optimization on the components at the same time. Results of classical algorithms are referenced to reduce the number of candidate topologies and greatly simplify the calculation process. Some calculation strategies, such as elitist strategy and clearing niche method, are adopted during optimization to make sure that the algorithm can convergence to global optimal result. Simulation results show that the new method has advantages over designing complex impedance matching network.
\end{abstract}

Keywords: transduce, impedance matching

\section{Introduction}

Piezoelectric transducers have been widely applied in different fields [1][2]. Most of traditional piezoelectric transducers are narrowband which can only produce large scale vibration within a narrow range of resonant frequency. However more and more applications need transducers to work within rather wide range. Similar to narrowband transducers, the equivalent impedance of broadband transducers is capacitive too. In most cases, the excitation source and the transducer are impedance mismatched. If no electrical impedance matching network (EIMN) is adopted, the power reflected from the transducer will reduce the efficiency of the source and, as the condition worsens, even cause permanent damage to components. For narrowband piezoelectric transducer, a suitable inductance would be enough in EIMN. As for a broadband piezoelectric transducer (BPT), however, methods of designing EIMN are usually complicated and hard to understand.

Some successful methods [3][4] of designing EIMN have been developed, but it is still meaningful to find a new way that is easy to realize in practice and has good performance at the same time. In this paper, a brief review about methods of designing EIMN is made first. Second, a method based on genetic algorithm (GA) developed in this paper is described in detail. Third, simulation results of several examples are given to confirm the effectiveness of the method. Last, this paper is summarized briefly.

A general purpose of designing EIMN is to make the signal's energy transfer from source to load as much as possible within a broad frequency range. It is realized by means of inserting a lossless two ports matching network between the source and the load as is shown in Fig. 1.

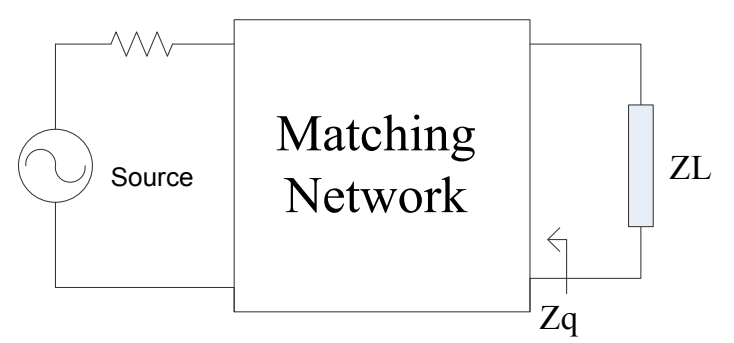

Fig. 1. The matching network is inserted between the source and load

The performance of EIMN in Fig. 1 can be evaluated by a parameter called transducer power gain (TPG) which is defined by Eq.(1).

$$
T P G=\frac{4 R_{Q} R_{L}}{\left(R_{Q}+R_{L}\right)^{2}+\left(X_{Q}+X_{L}\right)^{2}}
$$

In Equation (1), $\mathrm{ZL}=\mathrm{RL}+\mathrm{j} X \mathrm{~L}$ and $\mathrm{ZQ}=\mathrm{RQ}+\mathrm{jXQ} . \mathrm{ZQ}$ is the impedance seen from $Z \mathrm{~L}$ to the matching network. Through simple calculation, the value of TPG is between 0 and 1. A common purpose of the matching network is to make the TPG as big as possible within a specified frequency range.

There are mainly two types of broadband impedance matching methods: analytical method and CAD method. The analytical method started from Bode's theoretical research about impedance matching [5]. Fano developed Bode's theory and solved matching problem of any kind of load in a more general way Fano and Youla reconsidered this problem and made a progress[6][7]. Although analytical method can 
solve any impedance matching problem if the analytical form of the load is known, the method is very complicated. Besides, for most of the time, the analytical form of the load is unknown. These shortcomings limit the theory's practice in engineering.

In order to overcome the limitations of analytical theory, Carlin first developed the CAD method, namely real frequency technique (RFT), for solving single matching problem [4]. Yarman proposed simplified real frequency technique (SRFT) [8]. Both RFT and SRFT have been widely adopted in designing broadband EIMN [9][10]. Dedbeu has pointed out that an EIMN that have resonant units might have better performance than an EIMN of LC ladder structure, but it is not mentioned that how many resonant units are needed and the locations of the resonant units in network are determined by a little arbitrary way [11].
A different CAD method is recursive stochastic equalization (RSE) method [12]. Based on a topology imposed first, TPG is obtained as the objective function which has a function relationship with frequency and the impedance of the matching network. However, it is difficult to decide what kind of topology is appropriate especially when the topology of EIMN is complex.

From the above discussion, it can be concluded that EIMN synthesized by current typical methods still might have room for improvement. This paper puts forward to a method based on GA. This method can find the optimal topology as well as the values of the components automatically within a set of predefined topology. The definition of the set is very important to the success of the algorithm and is selected based on the experiences of current methods.

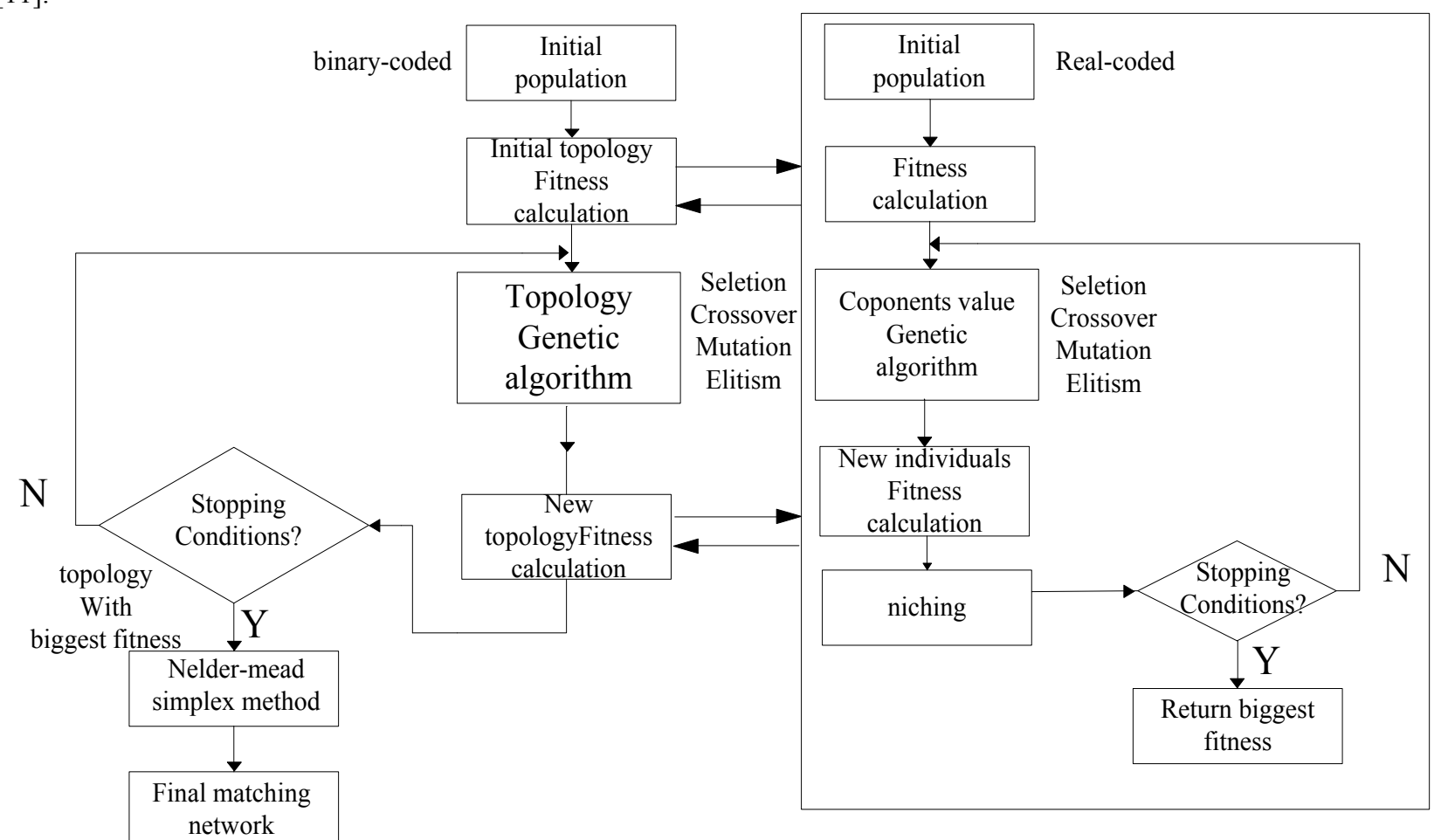

Fig. 2 Overview of the new method based on GA

\section{Design of Broadband EIMN Based On GA}

\subsection{Overview of the algorithm}

The idea of the GA-based method presented here is to find a way that not only both the network topology and the values of components can be optimized but also a lot of computing resources are not necessary so that the algorithm can run on a laptop. To reduce the computing resources and make sure the performance is good at the same time, prior experiences of classical methods are referenced to limit the number of the candidate topologies.

The calcullation process of the method is shown in Fig. 2 The topology optimization and the optimization of components are separated. Binary-coded GA is adopted to evolve the topology and real-coded GA is followed to determine the values of components and the TPG of the topology.

To improve the ratio of validity of new topology and reduce computation time, the matching network is assumed to have ladder-like structure that is shown in Fig 3. The types of $\mathrm{Z} 1, \mathrm{Z} 2, \ldots \mathrm{Zn}$ could be one of the four options which are L, C, parallel LC and serial LC and they are determined automatically by the algorithm.

The reason why the ladder-like structure is chosen is that it has been proved to be effective for most EIMN problems and some popular methods are based on this structure [5][6]. Another reason is that it can assure that most of the new networks generated by the algorithm are valid and can be easily evaluated which can reduce the computation time.

The number of branches in the structure is pre-specified, but it is not determined arbitrarily. The way the number is chosen is based on the conclusion that, for a specified load, if a matching network with LC ladder structure is applied, there will be a critical number of the branches. When the number of branches is less than the critical value, the more branches are, the better the performance of the EIMN is, and when it outnumbers the value, the performance is nearly invariable or even declines as the number increases. The critical number of branches of the LC ladder structure is 
specified to be the number of $\mathrm{Zn}$ in our algorithm. In part 3, an example will be used to prove that it is a feasible idea.

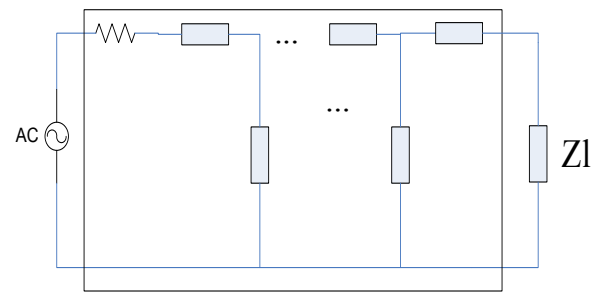

(a)

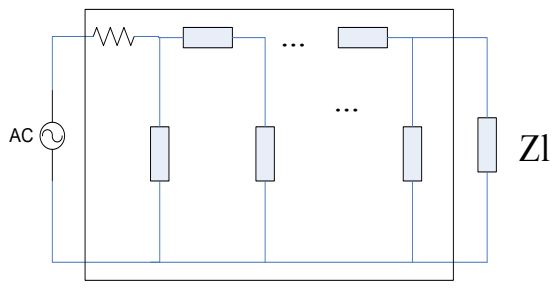

(b)

Fig. 3 (a)Ttype of matching network,and(b) $\pi$ type of matching network

\subsection{Topology optimization method}

In the algorithm, the matching network topologies are binary-encoded. Each branch is represented using two binary bits which is shown below.

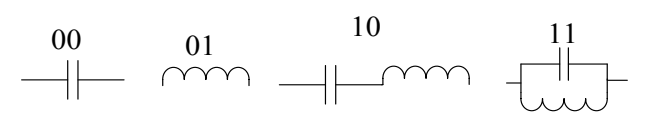

The fitness function we use here is shown in Eq. (2) in which TPG $(\omega)$ is shown in Eq. (1).

$$
\text { fitness }=\min (T P G(\omega))
$$

Each topology of the initial population is created randomly and the population size we use here is 120 .

Parents are selected by tournament [13] in three randomly-chosen individuals.

Uniform crossover [13] is used as crossover operator by which each gene of new individual is randomly chosen in two parents.

The probability of mutation [13] we use here is 0.1 .

The evolution will stop when the generation exceeds the maximum value $g_{\max }$ or a fitness value is bigger than a specified figure.

The new individual generated in each generation is different from all individuals in the population. Elitist strategy [13] is adopted that the individual with the best fitness in current generation will be reproduced to the next generation directly.

\subsection{Values optimization method}

To calculate the fitness of a topology, the components of the topology need to be determined first. A real-coded genetic algorithm (RCGA) [14] is adopted to perform optimization on the values of components. Although based on the same theory, some operators in RCGA are different from those in BCGA. A niching method [15] and the elitist strategy are adopted in combination to make sure the algorithm can find the global optimal solution of the multimodal fitness function.

In a practical transducer excitation circuit, a pulse transformer is needed. The effect of the transformer is: changing voltage, transforming impedance and blocking DC. In this method, the parameter of the transformer is determined by means of finding the optimal source resistance $\mathrm{Rg}^{\prime}$. The relation between $\mathrm{Rg}^{\prime}$ and real source resistance $\mathrm{Rg}$ is : $\mathrm{Rg}^{\prime}=\mathrm{n}^{2} \times \mathrm{Rg}$. The parameter $\mathrm{n}$ is realized by a transformer.

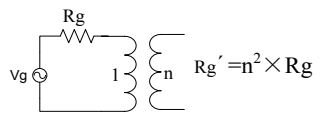

When GA is applied to optimize a multimodal function, it tends to converge to the local optimal values. To overcome this shortcoming, a clearing niching method is adopted [15]. In this method, the distance between two individuals is defined first which is the Euclidean distance in our algorithm. The capacity of a niche is defined which means that within the predefined range of an individual, only a limited number of individuals that have bigger fitness than others are effective, while other individuals' fitness are cleared to zero.

TPG is also used as the fitness of RCGA. When the algorithm finishes evolution, the genes in the chromosome that has the biggest fitness is assigned to the components of the specified topology.

Another shortcoming of GA is that the efficiency will decrease dramatically and be difficult to achieve the optimal solution when the algorithm is close to the optimal value. In order to overcome this problem, a local optimization algorithm called the Nelder-Mead simplex algorithm (NMSA) [16] is adopted as a complement to RCGA.

\section{Simulation and Test}

In this part, a typical example is presented to prove the effectiveness of the algorithm.

\subsection{EIMN of a piezoelectric ultrasound transducer}

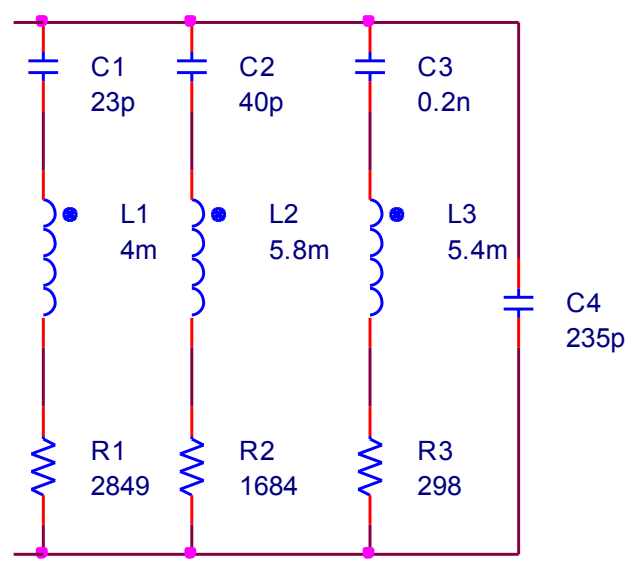

Fig. 4. Electro-mechanical equivalent circuit of a multi-resonant broadband piezoelectric ultrasound transducer

In this part, a broadband EIMN of a piezoelectric transducer is designed. Although the algorithm can be implemented directly with the measured data of the transducer, the analytical form of the impedance is still 
necessary for conveniently describing the design method process. A typical electro-mechanical equivalent circuit of a multi-resonant piezoelectric ultrasound transducer [5], as is shown in Fig. 4, is chosen to be matched.

The transducer works within the range of $200 \mathrm{KHz}-$ $600 \mathrm{KHz}$. As is shown in Fig. 5, there are three local maximum values at $196 \mathrm{KHz}, 366 \mathrm{KHz}$ and $570 \mathrm{KHz}$ which is $17.1 \mathrm{kohm}, 2.9 \mathrm{kohm}$ and $1.7 \mathrm{kohm}$ respectively. Each local maximum value is caused by one of the three LCR serial branches in Fig. 4.

Generally speaking, design of EIMN with good performance would be more difficult if the impedance of the transducer changes dramatically within its working frequency range. The impedance of the equivalent circuit decreases dramatically within the range of $200 \mathrm{KHz}-$ $250 \mathrm{KHz}$. The EIMN is designed within the range of $200 \mathrm{KHz}-600 \mathrm{KHz}$.

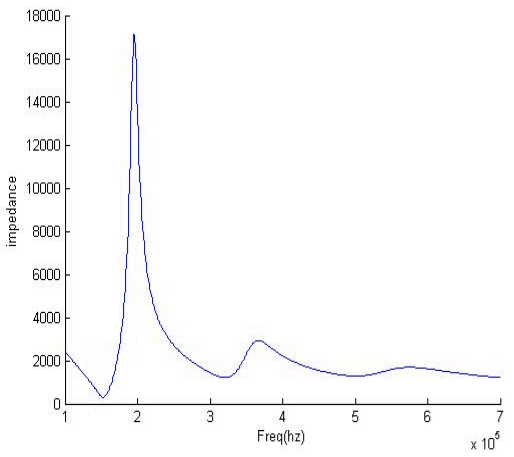

(a)

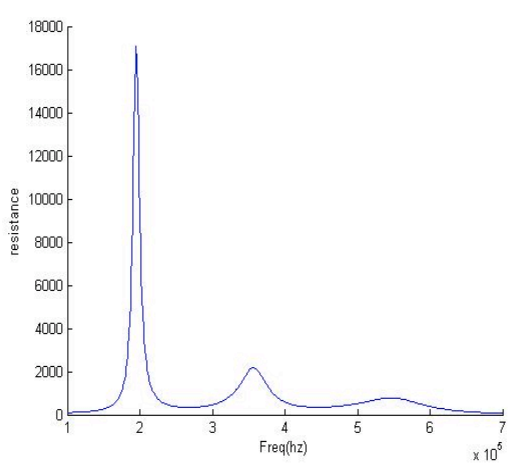

(b)

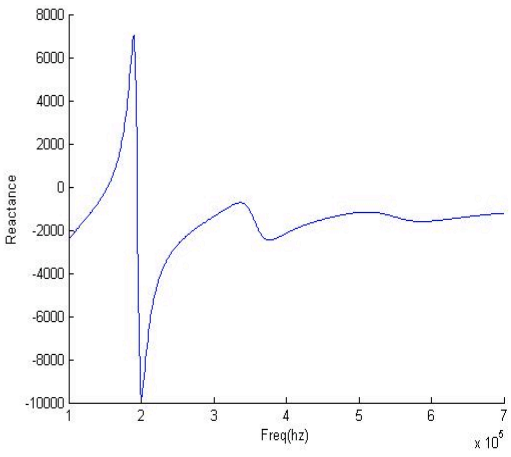

(c)

Fig. 5. Impedance characteristics of the equivalent circuit. (a) impedance (b) resistance (c) reactance

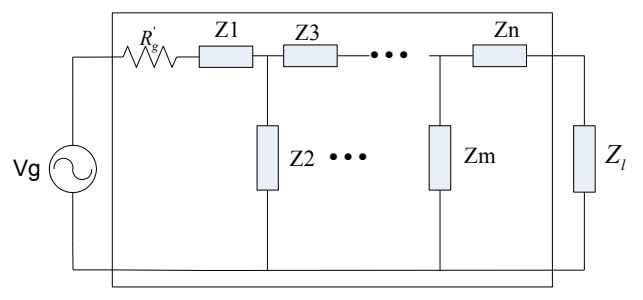

(a)

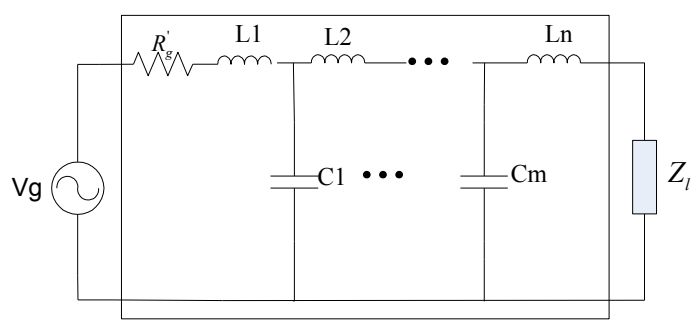

(b)

Fig. 6 Initial EIMN topology of the transducer; (b) LC ladder structure used for determining the number of $\mathrm{n}$

According to the algorithm process in second part, the number of branches in Fig. 6(a) needs to be specified first. The EIMN with LC ladder structure in Fig. 6(b) is used to determine the number of branches $n$ in Fig. 6(a).

Table. 1 Tmin of the EIMN in Fig. 6(b) with the number of branches

\begin{tabular}{ccccc}
\hline $\mathrm{n}$ & 3 & 5 & 7 & 9 \\
\hline $\mathrm{Tmi}$ & 0.36 & 0.43 & 0.46 & 0.42 \\
$\mathrm{n}$ & & 5 & 5 & 5 \\
\hline
\end{tabular}

As is shown in Table 1, Tmin of the EIMN in Fig. 6(b) changes with the number of branches. RCGA is adopted to specify the optimal components values in Fig. 6(b). It is appropriate that the number of branches in Fig. 6(b) is chosen to be three or five. Tmin is chosen to be the parameter during optimization. The EIMNs with three branches is shown in Fig .7 and the TPG within the operating band is shown in Fig .8. For ease of comparison, the TPG of the EIMN with LC ladder structure that is design by RFT is also shown in Fig. 8 .

As can be seen from Fig. 8, the Tmin of the EIMN with LC ladder structure is much lower than the EIMNs in Fig. 7 at the range of $350 \mathrm{KHz}-450 \mathrm{KHz}$.

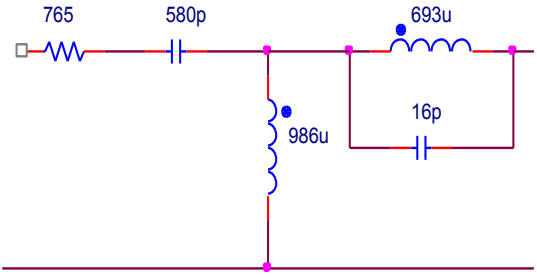

(a)

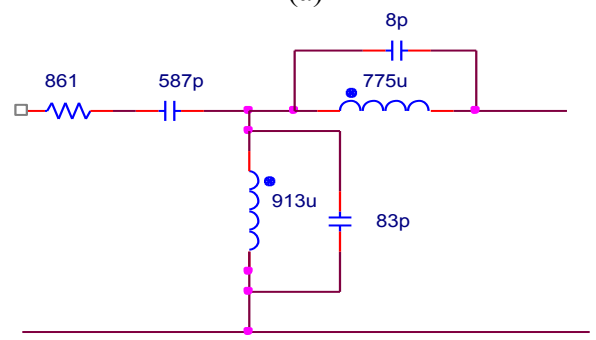

(b)

Fig.7 The EIMN with three branches designed by the method in the second part

Similar to analysis above, the EIMNs with five branches is also calculated and the results are shown in Fig. 9. Their 
TPG and the TPG of the EIMN with LC ladder structure are all shown in Fig .10. Although more components are used, the performance of the EIMNs with five branches doesn't get corresponding improvement Compared to the results of three branches.

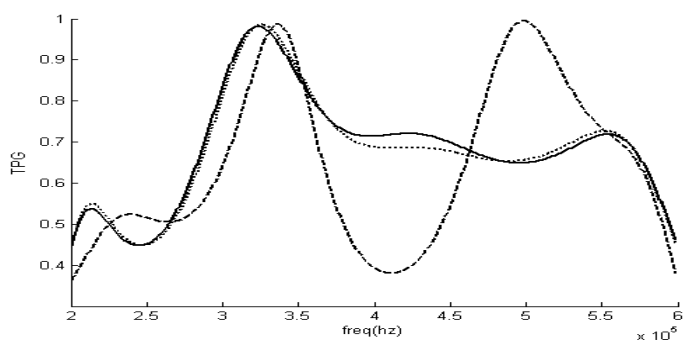

Fig.8 The EIMN with three branches designed by the method in the second part

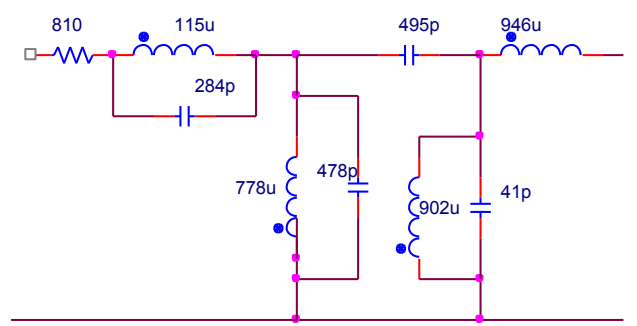

(a)

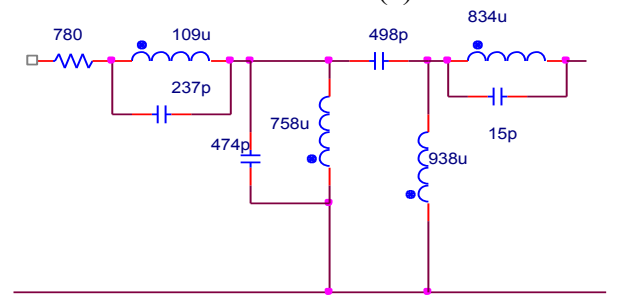

(b)

Fig.9 The EIMN with five branches designed by the method in the second part

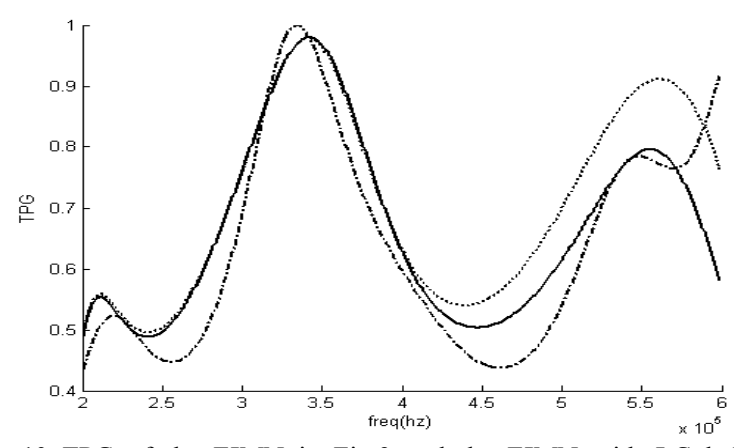

Fig.10 TPG of the EIMN in Fig.9 and the EIMN with LC ladder structure

...Fig. 9.(a); ——Fig. 9.(b); --- LC ladder structure

\section{Conclusions}

In this paper, an improved method of designing broadband electrical impedance matching network for piezoelectric ultrasound transducer has been proposed. The method introduced here is based on Genetic Algorithm. It can automatically synthesize the topology of electrical impedance matching network with resonant units. In order to decrease the computational time, results of popular methods are referenced to limit the size of the set of candidate topologies. Both BCGA and RCGA are implemented to optimize the topology and the components values respectively. The results of simulation have shown that the minimum of TPG obtained by this method might have better performance than the topology with LC ladder structure.

\section{References}

1. Amir Manbachi; Richard S C Cobbold. Development and application of piezoelectric materials for ultrasound generation and detection. Ultrasound. 2011, 19, pp.187-196.

2. J. K. Van Velsor, L. Premkumar, G. Chehab and J.L. Rose. Measuring the Complex Modulus of Asphalt Concrete Using Ultrasonic Testing. Journal of Engineering Science and Technology Review. 2011, 4(2), pp.160-168.

3. Haiying Huang. Broadband electrical impedance matching for piezoelectric ultrasound transducers, IEEE Transactions on Ultrasonics, Ferroelectrics, and Frequency Control. 2011, 58, pp. 2699-2707.

4. Herbert J. Carlin. A new approach to gain-bandwidth problems. IEEE Transactions on Circuits and Systems. 1977, 24, 170 - 175.

5. H. M. Bode. Network Analysis and Feedback Amplifier Design. New York: D. Van Nostrand, USA. 1945; pp. 281.

6. RM Fano. Theoretical limitations on the broadband matching of arbitrary impedances, Technical Report. ELSEVIER, Cambridge, Mass. USA, 1950, 249, pp.57-83.

7. D. C. YOULA. A New Theory of Broad-band Matching, IEEE Transactions on Circuit Theory. 1964, 11, pp.30-50.

8. B.S. Yarman; HERBERT J. CARLIN. A Simplified "Real Frequency" Technique Applied to Broad-Band Multistage Microwave Amplifiers. IEEE Transactions on Microwave Theory and Techniques. 1982, 30, pp.2216-2222.

9. Guolin Sun; Rolf H. Jansen. Broadband Doherty Power Amplifier via Real Frequency Technique. IEEE Transactions on Microwave Theory and Techniques. 2012, 60, pp.99-111.
10. David Yu-Ting Wu; Farouk Mkadem; Slim Boumaiza. Design of a Broadband and Highly Efficient $45 \mathrm{~W}$ GaN Power Amplifier via Simplified Real Frequency Technique. Microwave Symposium Digest (MTT), 2010 IEEE MTT-S International, Anaheim, California, USA. 23-28 May 2010

11. Hervb Dedieu; Catherine Dehollain; Jaques Neirynck; Graham Rhodes. New broadband-matching circuit, INTERNATIONAL JOURNAL OF CIRCUIT THEORY AND APPLICATIONS, 1994, 22, pp.61-69.

12. H Dedieu; Catherine Dehollain; Jacques Neirynck; and Graham Rhodes. A new method for solving broadband matching problems, IEEE Transactions on Circuits and Systems I: Fundamental Theory and Applications. 1994, 41, pp.561 - 571.

13. Z Michalewicz. Genetic algorithms + data structures $=$ evolution programs. 3rd, rev. and extended ed. Springer: Berlin Heidelberg, German. 1999.

14. F.Herrera; M.Lozano; J.L.Verdegay. Tackling RealCoded Operators and Tools for Behavioural Analysis. Artificial Intelligence Review. 1998, 12, pp.265-319.

15. A Petrowski. A clearing procedure as a niching method for genetic algorithms. Proceedings of IEEE International Conference on Evolutionary Computation. Nagoya, Japan, 20-22 May 1996, pp.798 -803.

16. J A Nelder; R Mead. A Simplex Method for Function Minimization. Computer Journal. 1965, 7, pp.308-313. 